\title{
On Congestion-Aware Broadcasting in V2X Networks
}

\author{
Fatma Hrizi and Fethi Filali \\ EURECOM, Mobile Communications Department \\ 2229 route des Crêtes, BP 193 \\ F-06560 Sophia-Antipolis Cedex, France \\ Email: hrizi, filali@eurecom.fr
}

\begin{abstract}
Due to the dramatic increase in the number of accidents all over the world, a rapid emergence of wireless vehicular networks has been perceived in the last few years, particularly, aiming at improving safety and efficiency in the road. Such applications require broadcast communications to disseminate emergency information in brief delay for all the vehicles in the area of danger. Designing an efficient broadcast scheme is crucial. For this reason, a large variety of approaches have been proposed. In this paper, we present a survey on various efforts for designing suitable multi-hop message dissemination schemes for vehicular systems especially which have been focused on reducing channel congestion. Furthermore, we propose a comparison of message dissemination proposals. Their performance evaluation and their advantages and limitations are also highlighted.

Index Terms-wireless vehicular networks, broadcast, safety applications.
\end{abstract}

\section{INTRODUCTION}

In the last few years, wireless vehicular networks have received a lot of attention. These networks are a special kind of wireless networks where vehicles, equipped with a particular electronic device, can communicate with each other and with potential infrastructures. The existence of such networks opens the way for a large range of applications. We consider that two of the most important classes of such applications are safety and non-safety applications. Non-safety applications improve passenger comfort and traffic efficiency and optimize the route to a destination. It includes information retrieval, entertainment services and congestion control. On the other hand, safety applications increase the safety of passengers by exchanging safety relevant information. They are mainly devoted to traffic control and vehicle collision avoidance services. Typical road safety applications include accident notification messages and distribution of information on traffic road conditions. The premise that wireless vehicular communications can enhance road safety efficiency has led governments and private entities to support several national and international projects around the globe. Many works have been carried out in this wide research area. However, many research issues are still open such as efficient messages dissemination, network scalability, and broadcast reliability. These challenging problems are due to the inherent characteristics of such networks where network partitioning occurs frequently due to high nodes' mobility, making end-to-end communication often impossible. The aim of this paper is to provide an up-to-date comprehensive survey and taxonomy of congestion-aware broadcast techniques for vehicular communications. We focus basically on recent broadcast proposals to alleviate the problem of congestion in vehicular environment. Each protocol operation will be outlined and the performance evaluation of each analyzed. The remainder of this paper is structured as follows. Section 2 overviews relevant proposals for message dissemination. In Section 3, we provide a comprehensive comparison of these broadcast protocols. Finally, we conclude this paper and we provide an overview of our future works in Section 4.

\section{CONGESTION-AWARE BROADCAST}

It is mandatory to support effectively the broadcast transfer mode in vehicular environment since the nature of most of safety applications requires reliable and fast information dissemination. For example, when an accident occurs, a safety message must be delivered to all nodes which are near to the accident area in order to avoid other potential accidents and to guarantee driver security. In essence, this can be ensured by applying broadcast techniques. Flooding is the classical broadcast mechanism, where every node in the network, after receiving the broadcast message, retransmits it to its neighbors. This technique is very easy and simple to implement and has an excellent message delivery rate even with a high mobility of nodes. However, it may lead to a very serious problem, often well-known as the broadcast storm problem [1]. This problem is characterized by an increase in bandwidth consumption and may lead to high messages collision rate and channel congestion. Moreover, high MAC collisions reduce the message delivery rate and increase delivery latency. Hence, many mechanisms have been proposed to alleviate broadcast storm problems and particularly channel congestion problem while preserving the high probability of receiving emergency messages that flooding mechanism ensures. Most of these proposals focused on the use of one or a combination of:

- The optimal selection of the relay node responsible for re-broadcasting.

- The adjustment of the transmission power according to the network conditions.

- The use of implicit acknowledgment mechanism to ensure reliability. 


\section{A. Relay selection methods}

Several protocols addressed congestion problem aiming at enhancing message dissemination and to improve floodingbased approaches. Most of them focused on how to select the relay node that will be responsible for forwarding safety messages. There is a very large body of proposals that choose to select relay node according to the distance from the source in order to reduce channel occupation and redundancy. Nevertheless, there are other approaches to select the relay node. These different schemes can be further categorized into four classes: distance-based, cluster-based, benefit-based and channel-aware approaches. In this section, we present a detailed description of the relevant approaches that we found in the literature for each aforementioned class.

1) Distance-based Relay Selection: The distance-based broadcast scheme allows only a given number of nodes to be involved in relaying the safety message to decrease network congestion and end-to-end delay. These specific nodes are called as relay nodes and are elected according to the distance from the previous relay. Many studies attempted to tackle this issue of relay selection.

The TRAck DEtection protocol (TRADE) protocol, described in [2], classifies the neighboring vehicles into three groups according to their position in the road; same road-ahead, sameroad behind and different road. Then, the algorithm selects a few nodes from each group to forward safety messages; the farthest vehicles from same road-ahead and same roadbehind and all vehicles from different road. A time to live value has to be specified aiming at limiting message broadcast to a specific number of hops. A given vehicle calculates the angle between its vector of direction and its neighbor vector and compares the result to an angle threshold. In this way, it could determine the category to which belong its neighbors. The second proposed protocol in [2], namely Distance Defer Transmission protocol (DDT), sets a defer time for message transmission to be inversely proportional to the distance from the source. And during defer time, a vehicle has to determine, according to the GPS information included in the multiple received message, if most of its transmission area has been covered by its neighbors, if not the vehicle should retransmit the safety message. The message's time to live is used also to reduce the number of hops. The bandwidth utilization and the reachability are the metrics which have been introduced to evaluate the performance of these proposals for both urban and rear areas. The results of the comparison of the two proposals with traditional broadcast approach reveal an improvement of bandwidth utilization for both of the protocols. In terms of message reachability, both TRADE and DDT perform better than traditional broadcast. We conclude that contrary to TRADE, DDT uses implicit acknowledgment guarantying reliability. In fact, a node receiving copies of the original safety message from different neighbors, make sure that the safety information is delivered to the whole transmission area. Nevertheless, there is no specification of the propagation direction of safety messages. On the other hand, TRADE reduces redundancy and congestion by limiting the number of message retransmitters but the accuracy in neighbors' list determination and the difficulty of choosing the appropriate angle threshold remains a problem to resolve. Many other proposals focused on selecting the farthest neighbor as a relay by using, at the same time, some additional approaches as an enhancement. The following protocols are further discussed later: Optimized Dissemination of Alarm Messages (ODAM) [3], Smart Broadcast Algorithm (SBA) [4], the proposal of [5], Contention Based Dissemination (CBD) [6], TRRS and ETRRS [7], UMB [8], FB [9] and the protocol proposed in [10].

In [3], Benslimanne proposes a protocol, namely ODAM, to disseminate safety messages among vehicles aiming at reducing network congestion and, consequently, increasing reliability of messages reception. The proposed protocol's main contribution lies on restricting the broadcast of safety messages to only relay nodes which are elected from a critical zone. The relays are chosen as the furthest neighbors away from the sender in order to ensure the coverage of the greatest zone not yet covered by the transmitter node. After waiting a defer time (inversely proportional to the distance from the sender), a node, which has not received the same message, re-broadcasts it. The author reveals performance evaluation results comparing ODAM to DDT and another broadcast protocol. The results of the experiments, regarding the number of informed vehicles against the transmission range, show that ODAM is more reliable in all considered situations.

SBA [4] is another proposal based on the same idea as ODAM with some differences. The authors assume that the road is partitioned in non-overlapping sectors. According to the sender position information included in the broadcast message, a node try to determine to which sector it belongs and enter the rebroadcast phase. A contention procedure is enabled where the waiting time for each node is inversely proportional to the distance from the sender. The vehicle aborts re-broadcasting if it receives a copy of the message from behind otherwise it forwards it after the waiting time. Using OPNET, the authors present performance evaluation of SBA. The proposal has been compared to flooding approach and another broadcast protocol. The results of experiments prove a high reachability and minimum redundancy for SBA.

Also in [5], Briesemeister et al. propose an approach to distribute safety messages by adopting the same idea of selecting a waiting time inversely proportional to the distance from the previous sender. Obviously, vehicles that are in the border of source's transmission range are selected as relays and are responsible for forwarding safety messages. Furthermore, the authors suggest limiting the number of hops traversed by the packet to a specific threshold MaxHops. In simulation scenarios, the rate of informed vehicles has been measured varying the percentage of equipped vehicles. The results show that the proposed protocol performs better when using the undivided road model because of the importance of the traffic density.

In [6], the authors describe CBD which employ a mechanism 
of contention to select nodes that will be responsible for retransmissions. The farther node from the sender waits the lowest time before re-broadcasting the safety message. Additionally, the authors suggest to use the Distributed Fair Power Adjustment for Vehicular networks (D-FPAV) algorithm, that will be discussed later, to decrease the beacons load and to provide more throughput to safety messages. The network simulator NS-2 has been used where IEEE802.11p was implemented. The results of the experiments reveal that $\mathrm{CBD}$ is more reliable in terms of delay and receipt probability when it is used with D-FPAV.

So, it can be said that distance-based broadcast approaches are based, fundamentally, on a contention mechanism where the waiting time is inversely proportional to the distance from the sender. The main advantage of this scheme is to decrease the number of retransmissions which results in improving the bandwidth consumption. Moreover, the broadcast procedure is controlled by limiting the number of hops ([5]) or by specifying the geographic area or/and the direction of propagation where the safety information must be delivered ([3], [4], [6]). In the following, we introduce some approaches that are distance-based using additional mechanism in order to better alleviate congestion problems and to guarantee a reliable safety information reception.

Kim et al. [7] raise the problem of selecting the relay in low density traffic scenario. They provide a new algorithm named Time Reservation-based Relay Node Selecting Algorithm (TRRS). This proposal is distance-based. Moreover, the authors assume that a relay node cannot be guaranteed to be at the border of the sender's transmission range especially in case of low density traffic where there are no vehicles at the border. Accordingly, a potential relay node that is not at the border can wait the time which waits a border node. Then, it will not wait wastefully and it can directly re-broadcast the message. Furthermore, the authors present the Enhanced TRRS (ETRRS) proposal as an enhancement of TRRS. The results of simulation studies and comparisons of TRRS and ETRRS with other relay selection schemes demonstrate that the proposed approach has the lowest end-to-end delay and has low network traffic. Moreover, ETRRS achieves better performance than TRRS. We think that adding such mechanism to a distancebased approach may result in an enhancement in broadcast performance in case of low density traffic. However, there is a potential difficulty in determining the optimal parameters for the calculation of contention window.

In [8], the authors suggest Urban Multi-hop Protocol (UMB), which is designed mainly to address the broadcast storm problems, especially, hidden node problem. The main idea is to use Ready/Clear to broadcast (RTB/CTB) mechanism to enhance messages reception. Receiving a successful CTB, the source node sends the packet acknowledged by the receiver. The proposed protocol has been compared to two other flooding-based MAC protocols. In all scenarios, UMB shows the highest reliability in terms of percentage of success and channel occupancy. Hence, we find that the channel reservation and the acknowledgment introduced by RTB/CTB scheme may reduce traffic congestion and increase reliability in message reception. However, in high packets load scenarios, UMB presents a very low speed in packet dissemination due to the overhead introduced by the RTB/CTB approach. [9] suggests Fast Broadcast (FB) protocol, whose main idea is to reduce the number of retransmissions using a distance-based scheme with an estimated transmission range. Indeed, this new scheme is composed of two phases; on the one hand, the estimation phase in which vehicles exchange their location information and their estimate of the transmission range in order to estimate and to update their transmission range. On the other hand, the broadcast phase which is a distance-based approach. Accordingly, a vehicle receiving a message computes a distance-based backoff and depending on the estimated transmission range declared in the received message. To assess FB performance, a simulation study has been performed. The authors have compared their proposal with a distance-based protocol that does not make use of dynamically estimated transmission range. The simulation results show that the proposed algorithm achieves its main goal by having the minimum number of hops crossed and also in terms of number of collisions. We believe that FB provides a realistic and accurate transmission range computing resulting in reducing collisions and end-to-end delay. Yet, the exchange of Hello messages still poses a problem and may increase traffic congestion.

[10] considers the problem of network fragmentation and proposes a distance-based protocol with a technique to detect and alleviate this critical problem particularly for safety applications. In case of network partitioning, the last relay includes its position and identifier in a message and sends it periodically. If it receives the same message with additional entries corresponding to the position and the identifier of vehicles in the direction of dissemination, it re-broadcasts the message. The authors used NS-2 in their simulations. The proposal has been compared to UMB with respect to the number of equipped vehicles. The results prove it has the highest percentage in dense or sparse network. However, overcoming fragmentation lead to a higher cost regarding the time to deliver message to all vehicles especially in sparse network where network fragmentation occurs often. The main advantage of this proposal is that vehicles need to exchange additional messages (beacons) only when they detect a fragmentation problem which may provide lower overhead and may reduce congestion problem. Nevertheless, this protocol performs well only in sparse networks in case of fragmentation. Previous contributions, as shown earlier, are based on relay election according to the distance from the previous relay. And generally, the waiting time of a node is inversely proportional to this distance so the farthest node is elected to be the next relay node. However, Taha et al. in [11] introduce a new approach. They assume that the road is divided into adjacent and non-overlapping segments. The vehicle in the most dangerous situation (the closest segment to the sender) has the highest priority to re-broadcast the safety message. Moreover, the proposed protocol adopts RTB/CTB mechanism to achieve reliable message dissemination and to 
avoid the hidden terminal problem. The authors conducted a performance evaluation of the proposal using a Matlabbased program. A comparison of the proposed protocol with UMB and SBA has been performed. We deduce that using this approach the latency for vehicles in the danger zone is improved. Nevertheless, the performance assessment results show that the good performances of this protocol are shown only in case of a large number of segments.

2) Cluster-based Relay Selection: In this section, we present an overview of the proposals in the domain of clustering and group management for vehicular networks. Most of the applications in wireless vehicular networks imply the need of group structuring, especially, in case of safety applications, when an emergency event occurs, usually a number of vehicles, in a given geographical area, share a common interest in receiving safety information. In [12], the authors present a cluster-based protocol, named BROADCOMM designed to achieve reliability in emergency data dissemination. They suggest an approach to construct the cell infrastructure, which consists of a partition of the road, and a protocol to broadcast safety information. To create the cell infrastructure, vehicles exchange their position information and speed. More precisely, this phase is achieved in two steps. In the first step, the cell creation, nodes exchange their position using Hello messages and determine their cell number with respect to the position of the first node which initiates the cell creation. The second step is the cell relay selection, only nodes which belong to the cell are permitted to participate in this phase. The elected vehicle will then behave as a base station. A process of update and maintenance is performed periodically after the cell reflector selection. If an emergency event occurs, the source node broadcasts the safety message to its cell member. A cell reflector, receiving the message, multicasts it to other neighbor reflectors. After that, each cell reflector broadcasts the safety message to all cell members. The authors conduct simulations using a simple workload model. They also consider the endto-end delay and the routing load to be measured. Moreover, a comparison with DOLPHIN, a flooding-based approach, has been performed. The results of this comparison prove that BROADCOMM outperforms DOLPHIN. The advantage of this scheme is reducing the number of broadcasting nodes which results in attenuating redundancy. However, the difficulty in updating and maintaining the formed cells remains a major problem that must be considered. Furthermore, the choice of the period of update is very critical.

Another study has been carried out in [13] to suggest a distributed dynamic clustering algorithm. Both a backbone creation strategy and a forwarding scheme for the MAC layer are proposed in [13]. The former assumes that a vehicle can be in two possible states: normal or backbone vehicle. A normal vehicle initiates the backbone creation when it does not receive periodic beacons and elects itself as a backbone member. A contention phase, depending on the estimation of the distance from the backbone member, is then executed by neighboring vehicles and whose winner will be the next backbone member. A backbone creation process is then ini- tiated. The latter, called Dynamic Backbone Assisted MAC (DBA-MAC), defines two priority classes: backbone member with the highest priority and normal vehicle. Additionally, two MAC schemes are proposed; a backbone member receiving a message sends immediately an acknowledgment to the sender, waits a SIFS, and then re-broadcasts it. This is called the Fast Multi-hop Forwarding (FMF) scheme. However, if the acknowledgment is not received, the node leaves this scheme and enters the basic MAC scheme with a higher priority to backbone members. This scheme is used also by normal vehicles. If the vehicle is a backbone member the contention window is initialized to a low value. Conversely, if it is a normal vehicle the size of the contention window is inversely proportional to the distance from the sender. The performance of the proposal is evaluated by means of NS-2 Simulator. A comparison with three other proposals. In terms of end-to-end delay, the proposed approach outperforms other protocols even in high density scenarios. We think that the proposed protocol reduces the number of retransmissions and increases reliability with the use of acknowledgment. Moreover, we deduce an improvement of the end-to-end delay since no mechanism of backoff is used.

We also found a study [14] that proposes a Local Peer Group (LPG) architecture whose main idea is to organize vehicles on the road into groups called LPG. The authors introduced in [14] two LPG architecture alternatives: dynamic and stationary LPG. The stationary LPG approach uses location-based and well defined LPG area so LPG members dynamically change as vehicles move. On the other hand, the dynamic LPG approach is based on the radio coverage of the neighboring vehicles. They also further detailed two dynamic LPG organization schemes to form, update and maintain LPG groups: the group header based organization and the linked-ECH based organization. The former consists in partitioning the vehicles into groups and defining a group header for each group which is responsible for LPG maintenance while the latter is based on forming a broadcast tree with LPG groups where the relative ordering between vehicles groups is respected. May be the main advantage of the stationary LPG approach is the lack of messages' exchange. So, there is no need to perform LPG forming and updating. However, there is a difficulty in partitioning the road into LPG areas and in the choice of the size of each stationary LPG. In case of dynamic LPG, we deduce that the proposal reduces message redundancy and improves latency. Nevertheless, the high amount of exchanged messages to update LPG may be considered as a major problem. Also, the difficulty in setting the period of update must be considered.

3) Benefit-based Relay Selection: A new approach for message forwarding is studied by Eichler et al. in [15], [16] where a new protocol based on information differentiation is proposed. In fact, to maximize the benefit of the whole network, each node computes the benefit provided by each packet to all the adjacent nodes which depends on various parameters such as the distance to information source, the information type and quality, the driving direction, vehicle speed and road type, 
and finally message specific characteristics like message age and last broadcast and reception time. Obviously, the packet providing the highest benefit to all neighbors has the highest priority to be forwarded. Furthermore, a contention scheme is introduced and which depends on the estimated benefit of the message to broadcast. The authors evaluate the efficiencies of the proposal by means of CARISMA as a road traffic simulator and NS-2 as a network simulator. The simulation results show that the proposed scheme accomplishes its goal in improving the overall network benefit and reducing the overall data throughput. This new strategy may help to improve message dissemination reliability by increasing the global network benefit, at the same time it may decrease traffic congestion and heavy collisions. However, we believe that the most critical challenge to be tackled is to set the appropriate parameters to calculate the benefit function since this can influence the accuracy of messages' benefit estimation.

4) Channel-aware Relay Selection: The only work we could find in the open literature for this category is described in [17] where the authors propose a contention-based approach to disseminate safety message based on the theory model of the real wireless channel, called REAR. The main idea of this proposal is to select the propagation relay according to the estimated receipt probability. Obviously, a node is selected as a relay if its neighbors have the highest receipt probability. Every node can calculate the estimated receipt probability for its neighbors based on their position and their environment exchanged via beaconing. The propagation is limited to a specific direction included in the message to broadcast with the node's list of neighbors. The network simulator NS-2 has been used. Furthermore, REAR is compared with a location-based algorithm. All simulation results reveal that REAR outperforms the location-based scheme regarding reliability rate and broadcast packets number. However, in terms of latency, REAR has longer latency than locationbased algorithm. We think that by using this novel approach the reliability for reaching the whole risk zone may increase since messages' redundancy is minimized by selecting specific nodes to re-broadcast safety messages. On the other hand, this protocol presents a potential difficulty in the determination of the receipt probability. Moreover, the introduced overhead by beacons is not negligible and may produce serious congestion problems.

\section{B. Power adjustment methods}

Another important area which has been considered by many researchers in vehicular networks is the adjusting of transmission power according to vehicle environment and many other circumstances in order to reduce safety messages redundancy. In this section, we briefly look at some of power adjustment proposals found in the literature. There are protocols which are distributed and use beacons exchange to collect information from neighbors while other use only local information to adjust the power of transmission, we deal with the distributed ones. In [18], Reumerman et al. presents a distributed communication protocol for safety applications. The idea is based on restrictive flooding mechanism where each node receiving, within a period of time, a number of copies of a given message higher than a threshold (floodingThreshold ) does not re-broadcast it. The threshold depends on the number of surrounding nodes detected. Besides flooding-based mechanism, the authors propose another approach to increase the performance of the protocol which is the power control mechanism. In fact, the transmission power is dynamically calculated on the basis of feedback information gathered from the channel (Hello messages) and using the average path-loss information per neighbor. The authors present experimental data intended to show the reliability and effectiveness of the protocol. The simulation results show that with the progressive reduction of the transmission power the message redundancy and the reachability are improved. However, we believe that redundancy still not negligible due to the use of flooding-based approach.

In [19], Torent-Morreno et al. propose a distributed algorithm, D-FPAV, for adaptive transmit power adjustment. The protocol requires that every node collects the status information of all its neighbors to compute the appropriate transmission power level. Using these information, a vehicle sets its transmit power level to the minimum value to achieve fairness. This value must be under a specific threshold MaxBeaconingLoad. The simulation environment has been NS-2. The performance evaluation experiments show that D-FPAV achieves its design goal of fairness in terms of Channel Busy Time. Additionally, a prioritization of safety message over beacons is observed since the probability of correctly receiving safety message with using D-FPAV is higher than the reception probability in case of no power control. In the other hand, the beacons reception probability has the highest values only in the area where their information is most relevant. D-FPAV is used typically to reduce the beaconing load on the channel in case of saturation. In turn, the adjustment of the transmission power of beacons reduce the collisions and provide to safety messages more bandwidth. Nevertheless, we think that the exchange of beacons cannot be considered as negligible and may produce collisions and channel congestion.

Mittag et al. present in [20] an analysis of the D-FPAV algorithm and the trade-off between the overhead caused by beacons exchange, on the one hand, and the accuracy of the knowledge of the status information of the surrounding nodes on the other hand. Moreover, they suggest a power adjustment approach consisting on a Distributed Vehicle Density Estimation scheme (DVDE) and a Segment-based Power Adjustment for Vehicular environment (SPAV). The DVDE provides an approximation of the surrounding traffic density for each vehicle according to the information included in the received beacons. This approximation is used then by SPAV to adjust dynamically the transmission power of vehicles. Other suggestions focused on estimating the local density of vehicles by the means of analytical models using local information. Artimy suggests in [21] a scheme that allows vehicles to estimate their local density using the average vehicle speed and the fraction of vehicles that are stopped due to traffic 
congestion. According to this density estimate, each vehicle can adjust their transmission power using Dynamic Transmission Range Assignment (DTRA) algorithm. This later uses two analytical models to compute the transmission power for each vehicle. We deduce that traffic congestion is reduced since no additional messages exchange but the problem of the accuracy of the density estimate remains. We found also in the literature another proposal [22] that introduces an approach to estimate the local density using an analytical model and to adjust the transmission range of the node according to this estimate. To estimate the local density, a node calculates the number of its neighbors by listening to the radio channel and evaluating the distance from each neighbor which can be derived from the signal strength or a timing-differential signal approach. The main advantage of such approach is to maximize the broadcast coverage. Moreover, there is no extra messages exchange which helps to attenuate traffic congestion. On the other hand, we cannot neglect the problem of the difficulty of estimating the accurate local node density.

\section{Increasing broadcast reliability with implicit acknowledg- ment}

Reducing the number of node that re-broadcasts safety messages is a new approach suggested by [23] and [24] to reduce channel congestion. In [23], Biswas et al. introduce a floodingbased transmission strategy with an implicit acknowledgment, named Intelligent Broadcast with Implicit Acknowledgment (I-BIA). After starting the periodic transmission of safety messages, a vehicle which receives the same message from behind stops broadcasting because it deduces that at least one vehicle in the back has received that message and will be responsible for forwarding it. This may help to avoid redundancy and, at the same time, increase message reception rate. On the other hand, if the vehicle does not receive any message with the same identifier within a given period of time, then it keeps on forwarding the message assuming the responsibility of propagating safety information. A simulation analysis has been performed using NS-2. The protocol was compared to the Naive Broadcast (NV) protocol as the direction-aware flooding protocol. The results from these experiments show that combining both safety data prioritization and I-BIA approach may lead to reliable message dissemination.

In [24], the authors propose a novel message dissemination approach for the Emergency Electronic Brake Light (EEBL) application in vehicular networks, namely Cluster Based broadcast $(\mathrm{CB})$, based on I-BIA algorithm. In addition to the I-BIA algorithm, $C B$ assumes that after receiving the same message from rear and after stopping message forwarding, a vehicle has to sense persistently the channel for messages coming from behind for a predefined period of time as it supposes that the vehicle taking the responsibility may leave and it should take it back in this case. The protocol was compared to directional flooding scheme. The results show that the advantage of CB over directional flooding, with respect to collision percentage and average relative latency, is obvious when the background traffic is important. Overall, we believe that this approach may decrease the number of retransmitters resulting in limitation in messages redundancy. Nevertheless, the probability of collisions and messages redundancy is not negligible since the flooding nature of each one of the proposals.

\section{PROTOCOLS COMPARISONS}

In this section, we provide a brief comparison of the protocols discussed previously. The following criteria are used in our comparison: the technique used to limit the propagation of the broadcast message, the use of beacons, the knowledge of the geographic position of the vehicle and the position of its neighbors, the application of acknowledgment techniques, the use of infrastructures, the utilization of the 802.11p standard, memory requirement, redundancy, delivery rate and latency. Table 1 shows that most of the proposals use a specific technique to limit the propagation of broadcast messages which can be a geographic area or a counter (TTL) decremented when the number of hops traversed by the broadcast packet is incremented. Also, we can observe that most of the distance-based protocols are beaconless while all of the cluster-based approaches are beaconed. In general, the distance-based schemes do not need the knowledge of neighbors' position so the exchange of beacons is unnecessary. However, the cluster-based approaches are based on the information collected from neighbors in order to form and maintain the clusters. Therefore, beacons exchange is crucial. All protocols assume that vehicles obtain their geographical position using specific devices (for example GPS). Table 2 reveals that most of the distance-based protocols achieve a medium delivery rate because of the potential collisions that may occur, a medium redundancy since only one selected node is responsible for message re-broadcasting and a low latency as the message propagation delay is reduced to the waiting time of the contention phase. On the other hand, cluster-based proposals are characterized by lower latency than the distancebased approaches, a low redundancy due to the clustering organization and a medium delivery rate since the exchange of beacons may cause severe collisions and channel congestion. The protocols using RTB/CTB (UMB and the proposal of [11]) mechanism may suffer from high latency because of the additional delay that introduces this mechanism.

\section{CONCLUSION AND FUtURE WORKS}

In this paper, we have provided a brief overview of the ongoing researches for vehicular networks and especially in the area of broadcast communications. We, first, surveyed the approaches proposed for reliable safety messages dissemination addressing the problem of congestion. Then, we provided a qualitative comparison of them and, additionally, giving the advantages and drawbacks of each one of them. Mainly, a general observation which can be made from this survey leads us to the rather conclusion that broadcasting for vehicular safety applications still a challenging topic requiring innovative approaches. Our future works will address this 
problem and we plan to design a reliable multi-hop broadcast protocol for safety applications.

\section{ACKNOWLEDGMENT}

We acknowledge the partial financial support of the European Community under the FP7 iTETRIS Project Contract no. FP7-224644.

\section{REFERENCES}

[1] Y. -C. Tseng,S. -Y. Ni,Y. -Chen, and J. -P. Sheu, The Broadcast Storm Problem in a Mobile Ad Hoc Network, in Proc. of the 5th annual ACM/IEEE international conference on Mobile computing and networking. August 1999.

[2] M. Sun, W. Feng, T. Lai, K. Yamada, H. Okada, and K. Fujimura, GPSbased message broadcast for adaptive inter-vehicle communications, in Proc. of the 52nd Vehicular Technology Conference (IEEE VTS-Fall VTC 2000), May 2000.

[3] A. Benslimane, Optimized Dissemination of alarm Messages in Vehicular Ad-hoc Networks (VANET), in Proc. of the 7th IEEE International Conference on High Speed Networks and Multimedia Communications (HSNMC'04), 2004.

[4] E. Fasolo, R. Furiato, and A. Zanella, Smart Broadcast algorithm for inter-vehicular communications, in Proc. of the Wireless Personal Multimedia Communication (WPMC'05), IWS 2005, September 2005.

[5] L. Briesemeister, L. Schafers, and G. Hommel, Disseminating Messages among highly mobile hosts based on Intervehicle Communication, in Proc. of the IEEE Intelligent Vehicles Symposium, October 2000.

[6] M. Torrent-Moreno, Inter-Vehicle Communications: Assessing Information Dissemination under Safety Constraints, in Proc. of the 4th Annual IEEE/IFIP Conference on Wireless On Demand Network Systems and Services (WONS), January 2007.

[7] T. Kim, W. Hong, and H. Kim, An effective Multi-hop Broadcast in Vehicular Ad-hoc Network, in Proc. of the Architecture of Computing Systems (ARCS 2007), March 2007.

[8] G. Korkmaz, and E. Ekici, Urban Multi-hop Broadcast Protocol for InterVehicle Communication Systems, in Proc. of the 1st ACM workshop on vehicular Ad Hoc networks (VANET'04), October 2004.

[9] C. Plazzi, S. Ferretti, M. Roccetti, G. Pau, and M. Gerla, How Do You Quickly Choreograph Inter-Vehicular Communications? A Fast Vehicleto-Vehicle Multi-Hop Broadcast Algorithm, Explained, in Proc. of the 4th IEEE Consumer Communications and Networking Conference (CCNC 2007), January 2007.

[10] S. Khakbaz, and M. Fathy, A Reliable Method for Disseminating Safety Information in Vehicular Ad Hoc Networks Considering Fragmentation Problem, in Proc. of the Fourth International Conference on Wireless and Mobile Communications (icwme), 2008.

[11] M. Taha, and Y. Hasan, VANET-DSRC Protocol for Reliable Broadcasting of Life Safety Messages, in Proc. of the IEEE International Symposium on Signal Processing and Information Technology, December 2007.

[12] M. Durresi, A. Durresi, and L.Barolli, Emergency Broadcast Protocol for Inter-Vehicle Communications, in Proc. of the 11th International Conference on Parallel and Distributed Systems (ICPADS'05), 2005.

[13] L. Bononi, and M. Di Felice, A Cross Layered MAC and Clustering Scheme for Efficient Broadcast in VANETs, in Proc. of the IEEE International Conference on Mobile Ad hoc and Sensor Systems (MASS 2007), October 2007.

[14] W. Chen, and S. Cai, Dynamic Local Peer Group Organizations for Vehicle Communications, in Proc. of Vehicleto-Vehicle Communications Workshop (V2VCOM 2006) co-located with ACM MobiQuitous, July 2006.

[15] S. Eichler, C. Schroth, T. Kosch, and M. Strassberger, Strategies for Context-Adaptive Message Dissemination in Vehicular Ad Hoc Networks, in Proc. of the Second International Workshop on Vehicle-to-Vehicle Communications (V2VCOM), July 2006.

[16] S. Eichler, and C. Schroth, A Multi-Layer Approach for Improving the Scalability of Vehicular Ad-Hoc Networks, in Proc. of the Conference on Communication in Distributed Systems (KiVS 07), Workshop: 4th Workshop on Mobile Ad Hoc Networks (WMAN 2007), March 2007.

[17] H. Jiang, H. Guo, and L. Chen, Reliable and Efficient Alarm Message Routing in VANET, in Proc. of the 28th International Conference on Distributed Computing Systems Workshops, 2008.
[18] H. Reumerman, and M. Runi, Distributed Power Control for Reliable Broadcast in Inter-Vehicle Communication Systems, in Proc. of the 2nd International Workshop on Intelligent transportation (WIT 2005), March 2005.

[19] M. Torrrent-Moreno, P. Santi, and H. Hartenstein, Distributed Fair Transmit Power Adjustment for vehicular Ad Hoc Networks, in Proc. of the 3rd Annual IEEE Communications Society on Sensor and Ad Hoc Communications and Networks (SECON '06), September 2006.

[20] J. Mittag, F. Schmidt-Eisenlohr, M. Killat, J.Hrri, and H. Hartenstein, Analysis and design of effective and lowoverhead transmission power control for VANETs, in Proc. of the fifth ACM international workshop on Vehicular Inter-NETworking (VANET'08), September 2008.

[21] M. Artimy, Local Density Estimation and Dynamic Transmission-Range Assignment in Vehicular Ad Hoc Networks, in Proc. of the IEEE Transactions on Intelligent Transportation Systems, September 2007.

[22] X. Li, T. D.Nguyen, and R. P. Martin, Using Adaptive Range Control to Maximize 1-Hop Broadcast Coverage in Dense Wireless Networks, in Proc. of the First Annual IEEE Communications Society Conference on Sensor and Ad Hoc Communications and Networks (IEEE SECON), October 2004

[23] S. Biswas, M. Tatchikou, and F. Dion, Vehicle-to-Vehicle Wireless Communication protocols for Enhancing Highway Traffic Safety, in Proc. of the IEEE Communications Magazine, IEEE Publication Date: Jan.2006 Volume: 44, Issue: 1 pages: 74-82.

[24] S. Biswas, M. Tatchikou, and F. Dion, Wireless Local Danger Warning Using Inter-Vehicle Communications in Highway Scenarios, in Proc. of the 14th European Wireless Conference (EW 2008), June 2008. 
TABLE I

A COMPARISON OF MESSAGE DISSEMINATION PROTOCOLS 1

\begin{tabular}{|c|c|c|c|c|c|c|}
\hline Protocol & $\begin{array}{l}\text { Propagation Lim- } \\
\text { itation }\end{array}$ & Beacon & $\begin{array}{l}\text { Neighbors Posi- } \\
\text { tion }\end{array}$ & Self Position & ACK & $\begin{array}{l}\text { Infrastructure } \\
\text { Use }\end{array}$ \\
\hline TRADE & TTL & Beaconed & Yes & Yes & No & No \\
\hline DDT & TTL & Beaconless & $\begin{array}{l}\text { No/only source } \\
\text { position }\end{array}$ & Yes & Implicit ACK & No \\
\hline ODAM & Risk zone & Beaconless & $\begin{array}{l}\text { No/only source } \\
\text { position }\end{array}$ & Yes & No & No \\
\hline SBA & $\begin{array}{l}\text { Direction } \\
\text { propagation }\end{array}$ & Beaconless & $\begin{array}{l}\text { No/only source } \\
\text { position }\end{array}$ & Yes & No & $\mathrm{No}$ \\
\hline Prop. of [5] & Local area + TTL & Beaconless & $\begin{array}{l}\text { No/only source } \\
\text { position }\end{array}$ & Yes & No & No \\
\hline CBD & $\begin{array}{lr}\text { Specific } & \text { area } \\
\text { (rectangle } & \text { of } \\
2 \mathrm{KM}) & \end{array}$ & Beaconed & $\begin{array}{l}\text { Yes (maintain a } \\
\text { list) }\end{array}$ & Yes & No & No \\
\hline TRRS/ETRRS & Specific range & Beaconless & $\begin{array}{l}\text { No/only source } \\
\text { position }\end{array}$ & Yes & No & No \\
\hline UMB & $\begin{array}{l}\text { Direction of } \\
\text { propagation }\end{array}$ & Beaconless & $\begin{array}{l}\text { No/only } \\
\text { position }\end{array}$ & Yes & Yes & $\begin{array}{l}\text { Yes (for intersec- } \\
\text { tions) }\end{array}$ \\
\hline FB & Area of interest & Beaconed & Yes & Yes & No & No \\
\hline Prop. of [10] & $\begin{array}{l}\text { Direction of } \\
\text { propagation }\end{array}$ & $\begin{array}{l}\text { Beaconless (Only } \\
\text { in fragmentation } \\
\text { case) }\end{array}$ & $\begin{array}{l}\text { No/only source } \\
\text { position }\end{array}$ & Yes & No & No \\
\hline Prop. of [11] & Rectangle & Beaconless & $\begin{array}{l}\text { No/only source } \\
\text { position }\end{array}$ & Yes & Yes & No \\
\hline BROADCOMM & Not specified & Beaconed & Yes & Yes & No & No \\
\hline Prop. of [13] & $\begin{array}{l}\text { TTL + direction } \\
\text { of propagation + } \\
\text { risk zone }\end{array}$ & Beaconed & $\begin{array}{l}\text { No/only source } \\
\text { position }\end{array}$ & Yes & Yes & No \\
\hline REAR & $\begin{array}{l}\text { Direction of } \\
\text { propagation }+a \\
\text { risk zone }\end{array}$ & Beaconed & Yes & Yes & No & No \\
\hline
\end{tabular}

TABLE II

A COMPARISON OF MESSAGE DISSEMINATION PROTOCOLS 2

\begin{tabular}{|l|l|l|l|l|}
\hline Protocol & Memory requirement & Retransmissions & Delivery & Redundancy \\
\hline TRADE & Yes & No & Medium & High \\
\hline DDT & Yes & No & Medium & High \\
\hline ODAM & Yes & No & Medium & Medium \\
\hline SBA & Yes & Yes & Medium & Medium \\
\hline Prop. of [5] & Yes & No & Medium & Medium \\
\hline CBD & Yes & No & Medium & Medium \\
\hline TRRS/ETRRS & Yes & No & Medium & High (TRRS)/Lower( \\
& & & Low \\
\hline UMB & Yes & Yes & High & Medium \\
\hline FB & Yes & Yes & Medium & Medium \\
\hline Prop. of [10] & Yes & Yes & Medium & Medium \\
\hline Prop. of [11] & Not mentioned & Not mentioned & Medium & Medium \\
\hline BROADCOMM & Not mentioned & Not mentioned & High & Low \\
\hline Prop. of [13] & Yes & Yes & High & Low \\
\hline REAR & Yes & No & Medium & Low \\
\hline
\end{tabular}

